\title{
The Expansion Process in a Counter-flow Vortex Tube
}

\section{Yunpeng Xue*, Mehdi Jafarian, Amanullah Choudhry and Maziar Arjomandi}

School of Mechanical Engineering, the University of Adelaide South Australia 5005, Australia

\begin{abstract}
The process of temperature separation in a Ranque-Hilsch vortex tube is dependent on a multitude of factors, such as the pressure gradients, flow stagnation and mixture, energy transfer between different flow layers and heat transfer between the tube and the ambient air. The pressure gradient in an expansion process within a vortex tube has been proposed as the dominating reason for the temperature drop. However, at present, there is no general agreement within the research community for the expansion process itself, primarily due to the complexity of the internal flow conditions in the tube. Therefore, in the present article, a deeper insight into the separation mechanism within a vortex tube is presented, based on an analytical analysis using different expansion models, including isentropic expansion, free expansion and Joule-Thomson expansion. It was observed that the isentropic expansion is the only possible expansion process within a vortex tube. This was confirmed through comparison with experimental results obtained from different sources.
\end{abstract}

Keywords: Vortex tube; Expansion process; Isentropic expansion

\section{Introduction}

A Ranque-Hilsch vortex tube is a thermal device which generates two exhaust gases at different temperature from a single injection port. The typical structure of a counter-flow vortex tube and the proposed flow pattern within the tube are presented in Figure 1 [1]. The working medium (shown in yellow), generally air or other compressible gases, is injected into the tube through a tangential nozzle. The low temperature stream (shown in blue) is discharged through the central exit, near the injection port, and the high temperature stream (shown in red) is exhausted from the peripheral exit, at the far end of the tube. The perceived benefits of the temperature separation within a vortex tube, such as instant cold and hot streams of fluid, adjustable stream temperatures, reliability and low cost, make it an attractive device in many applications where such temperature separation is beneficial. These applications can include the use of vortex tube for cooling purposes [2,3], ice production [4], and mixture separation [5,6]. Since its invention, in 1933 [7], investigations have been carried out to understand the processes involved in a vortex tube. Much of the earlier research in the subject matter has primarily focussed on the separating mechanisms within the tube, including theoretical analysis of the separation process [8,9], optimisation of the tube performance for different applications [10,11], numerical simulations of the temperature separation within the tube [12-15] and investigation of the internal flow behaviour [1,16-18]. Different understandings of the internal flow mechanisms and explanations for the energy separation

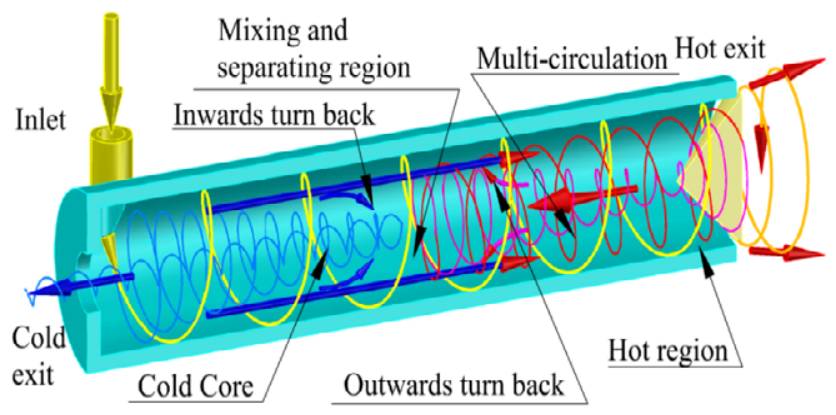

Figure 1: The typical structure of a counter-flow vortex tube and its hypothesized flow pattern within the tube. within the vortex tube, which have been reviewed and analysed in recent publications [19-22], imply the lack of general consensus and the requirement of clarification of the complex separating phenomenon. The internal pressure gradient has been proposed as the dominating factor for the energy separation phenomenon within a vortex tube [14,23-26]. Ranque proposed that compression and expansion effects are the main reasons for the temperature separation in the tube $[7,27]$. In a recent research, the adiabatic expansion and compression caused by turbulent eddies was stated as the reason for the temperature drop and the temperature rise in a vortex tube, respectively [28]. However, the proposal regarding the compression inside a vortex tube is not particularly convincing, primarily due to the internal pressure profile, as discussed in previous research $[1,22]$. On the other hand, a general agreement regarding the expansion process with the tube as being the dominating factor for the temperature drop has also been observed in literature $[1,14,25,26,29]$. It has, furthermore, been reported that the cooling performance of a vortex tube depends on the properties of the working gases. In terms of the cooling performance, argon and helium have been found to have a better performance than any other gas, including carbon dioxide, air, ammonia, nitrogen, oxygen and water vapour [30,31]. A previous numerical investigation on the cooling effect of a vortex tube, considering carbon dioxide, air, helium, ammonia, nitrogen, oxygen and water vapour, reported the agreement of the helium's best cooling effect [32]. The maximum value of specific heat capacity ratio and minimum molecular weight of helium was stated as the reason for its superior cooling performance. However, this explanation is not convincing, due to the significantly different tube performance from other publications [30,31]. Moreover, the results were achieved based on inappropriate parameters, i.e. the specific heat capacity ratio and the inlet velocity. In 2013, a comparison of the vortex

*Corresponding author: Yunpeng Xue, School of Mechanical Engineering, the University of Adelaide South Australia 5005 and Australia, Tel: +61 (0)8 8313 2577; Fax: +61 (0)8 8313 4367; E-mail: yunpeng.xue@adelaide.edu.au

Received March 13, 2015; Accepted March 24, 2015; Published March 31, 2015

Citation: Xue Y, Jafarian M, Choudhry A, Arjomandi M (2015) The Expansion Process in a Counter-flow Vortex Tube. JF I u i d $\mathrm{M} \mathrm{e} \mathrm{c} h$ O p e $\mathrm{n}$ A C C 2: 114. doi:10.4172/2090-8369.1000114

Copyright: () 2015 Xue Y, et al. This is an open-access article distributed under the terms of the Creative Commons Attribution License, which permits unrestricted use, distribution, and reproduction in any medium, provided the original author and source are credited. 
tube performance using different gases, i.e., nitrogen, carbon dioxide, $\mathrm{CH}_{2} \mathrm{~F}_{2}, \mathrm{CHClF}_{2}, \mathrm{C}_{2} \mathrm{H}_{2} \mathrm{~F}_{4}$, and $\mathrm{C}_{2} \mathrm{H}_{5} \mathrm{~F}$, was performed, in which the JouleThomson expansion was reported as the dominating expansion model within the vortex tube [33]. However, interestingly, the employment of $\mathrm{CHClF}_{2}, \mathrm{C}_{2} \mathrm{H}_{2} \mathrm{~F}_{4}$, and $\mathrm{C}_{2} \mathrm{H}_{5} \mathrm{~F}$ in the vortex tube did not result in the energy separation phenomenon. Instead, the temperature of the fluid at both the exits was observed to drop. This different performance implies the unclear nature of the expansion process in a vortex tube and prompts further research in the phenomenon. Therefore, in order to explain the expansion process in a vortex tube, this paper reports the findings from an analytical investigation of the cooling performance of different adiabatic expansion models. For a given set of conditions, estimates of the resultant cooling from the different models, including the isentropic expansion model, the free expansion model and the Joule-Thomson expansion model, are computed and compared with experimental results of a vortex tube system under similar conditions. The model that best predicts the overall cooling can be considered as the governing expansion mechanism within the tube. Furthermore, to investigate the effect of different expansion rates on the tube performance, the paper explores the effects of different working fluids on the expansion process. It has generally been observed that the working gases, within a tube, expand differently, due to their dissimilar properties. This difference principally indicates the significant impact of the gas property on both the temperature variation in the expansion process, as well as the cooling performance of the vortex tube $[30,34-$ 38]. Based on the diverse cooling performance of a vortex tube through the use of different working fluids, further analysis of the expansion process is conducted to identify the impact of the fluid property and hence to contribute to the understanding of the expansion process.

\section{Expansion Models}

To enable the comparison between the cooling capability of the different models and that of a vortex tube, a simplified model of the cooling part of a vortex tube is selected in this analysis. It is assumed that pressurised gas is injected into an isolated cavity and then exhausted after undergoing the different expansion processes. The temperature variation of the exhaust fluid as a function of its initial condition is then compared with the average temperature drop from vortex tube systems under similar conditions. The models tested in the present research are described further, in brevity, as follows

\section{The isentropic expansion model}

In an adiabatic and isentropic expansion process, the variations in temperature of a real gas, $T$, pressure, $P$, and entropy, $S$, can be related by:

$$
\left(\frac{\partial T}{\partial P}\right)_{S}=-\frac{\left(\frac{\partial S}{\partial P}\right)_{T}}{\left(\frac{\partial S}{\partial T}\right)_{P}} .
$$

From Maxwell's equation, the variation in entropy with pressure at a constant temperature is given by the following expression:

$$
\left(\frac{\partial S}{\partial P}\right)_{T}=-\left(\frac{\partial V}{\partial T}\right)_{P}=-\alpha V .
$$

Here, $\alpha$ is the volume expansivity and $V$ is the gas volume. On the other hand, the variation in entropy with the temperature at a constant pressure is given by:

$$
\left(\frac{\partial S}{\partial T}\right)_{P}=\frac{C_{P}}{T}
$$

Therefore, equation (1) can be rewritten as:

$$
\left(\frac{\partial T}{\partial P}\right)_{S}=-\frac{\left(\frac{\partial S}{\partial P}\right)_{T}}{\left(\frac{\partial S}{\partial T}\right)_{P}}=\frac{\alpha V T}{C_{P}} .
$$

To enable a quantitative analysis of the temperature variation in the expansion process, the Virial equation of state can be used in this analysis [39]:

$$
P V=A+B P+C P^{2}+\cdots
$$

Here, A, B, C, are Virial coefficients and are dependent on the temperature only. Substituting the Virial equation into equation 3 , and replacing $A=R T$ and $B=b-\frac{a}{R T}$, the following relationship can be
derived:

$$
\left(\frac{\partial T}{\partial P}\right)_{S}=\frac{1}{C_{p}}\left(\frac{R T}{P}-\frac{a}{R T}\right) .
$$

Here, $a$ and $b$ are coefficients of the Van der Waals equation of state, $R$ is the specific gas constant, $P$ is the pressure. Therefore, the equation describes the rate of temperature change with pressure as a function of initial conditions of the simplified system in an isentropic expansion.

\section{The free expansion model}

In a free expansion, the internal energy of a system stays constant during the process; hence $d U=0$. Therefore, the temperature of an ideal gas in a free expansion stays constant; while, for a real gas, depending on the initial conditions, the temperature changes. Similar to an isentropic expansion, the relationship between the temperature, pressure and internal energy in a free expansion process can be written as:

$$
\left(\frac{\partial T}{\partial P}\right)_{U}=-\frac{\left(\frac{\partial U}{\partial P}\right)_{T}}{\left(\frac{\partial U}{\partial T}\right)_{P}} .
$$

Furthermore, the internal energy of the system can be expressed as a function of enthalpy, volume and pressure.

$$
. U=H-P V
$$

The derivative of this function with respect to the pressure at a constant temperature can be written as follows:

$$
\left(\frac{\partial U}{\partial P}\right)_{T}=\left(\frac{\partial H}{\partial P}\right)_{T}-\left(\frac{\partial P V}{\partial P}\right)_{T}=\left(\frac{\partial H}{\partial P}\right)_{T}-V-P\left(\frac{\partial V}{\partial P}\right)_{T} .
$$

The enthalpy of the system can be related to the physical properties of the gas by [39]:

$$
\left(\frac{\partial H}{\partial P}\right)_{T}=V-T\left(\frac{\partial V}{\partial T}\right)_{P}
$$

Therefore,

$$
\left(\frac{\partial U}{\partial P}\right)_{T}=-T\left(\frac{\partial V}{\partial T}\right)_{P}-P\left(\frac{\partial V}{\partial P}\right)_{T}
$$

At a constant pressure, the variation of the internal energy with temperature can be expressed by: 
$\left(\frac{\partial U}{\partial T}\right)_{P}=\left(\frac{\partial H}{\partial T}\right)_{P}-\left(\frac{\partial P V}{\partial T}\right)_{P}=C_{P}-P\left(\frac{\partial V}{\partial T}\right)_{P}$

Combining equation 8 and 9, gives:

$$
\left(\frac{\partial T}{\partial P}\right)_{U}=\frac{-T\left(\frac{\partial V}{\partial T}\right)_{P}-P\left(\frac{\partial V}{\partial P}\right)_{T}}{C_{P}-P\left(\frac{\partial V}{\partial T}\right)_{P}} .
$$

Substituting the Virial equation into equation 10 and taking, $A=R T, B=b-\frac{a}{R T}$ the following relationship can be derived:

$$
\left(\frac{\partial T}{\partial P}\right)_{U}=\frac{a T}{C_{P} R T^{2}-R^{2} T^{2}-P a} .
$$

Equation 11 depicts the free expansion model where the temperature variation with pressure at a constant energy level is related to the properties of the fluid.

\section{The Joule-Thomson expansion model}

In a Joule-Thomson expansion process, the relationship between the temperature and pressure can be expressed as:

$$
\left(\frac{\partial T}{\partial P}\right)_{H}=-\frac{\left(\frac{\partial H}{\partial P}\right)_{T}}{\left(\frac{\partial H}{\partial T}\right)_{P}}=-\frac{\left(\frac{\partial H}{\partial P}\right)_{T}}{C_{P}} .
$$

Using equation 7, equation 12 can be rewritten as:

$$
\left(\frac{\partial T}{\partial P}\right)_{H}=\frac{T\left(\frac{\partial V}{\partial T}\right)_{P}-V}{C_{P}}=\frac{\alpha T V-V}{C_{P}} .
$$

Substituting the Virial equation and taking, $A=R T, B=b-\frac{a}{R T}$ the temperature variation in a Joule-Thomson expansion can be expressed by the following equation:

$$
\left(\frac{\partial T}{\partial P}\right)_{H}=\frac{1}{C_{P}}\left(\frac{2 a}{R T}-b\right) .
$$

The quantitative temperature difference between the isentropic and constant enthalpy (Joule-Thomson) expansion process is:

$$
\left(\frac{\partial T}{\partial P}\right)_{S}-\left(\frac{\partial T}{\partial P}\right)_{H}=\frac{V T \alpha}{C_{P}}-\frac{V(T \alpha-1)}{C_{P}}=\frac{V}{C_{P}}>0 .
$$

Since the ratio of specific volume to the specific heat capacity at constant pressure is always positive, the temperature variation in an isentropic process is always greater than that in a Joule-Thomson process. In other words, if all other conditions in the process are kept similar, the cooling capability of an isentropic expansion is always greater than that of a Joule-Thomson expansion.

\section{Comparison of the Expansion Models}

The nature of the expansion within a vortex tube can be determined by comparing its cooling capability to the cooling predictions of different expansion models. A quantitative analysis of the temperature variation during different expansion processes can be achieved using equation 4,11 and 14. The comparison of the expansion models can help elucidate the expansion process within the Ranque-Hilsch vortex tube. For the purpose of calculations, the initial and final pressures of the system are set as 2 bar and 1 bar, respectively; whereas, the initial temperature of the gas is set to $290 \mathrm{~K}$. These initial conditions have been selected in order to facilitate comparisons with the actual maximum temperature drops from a vortex tube operating at the similar conditions, as reported in [30,40], including the inlet pressure, inlet temperature and outlet pressure. The temperature profiles in different expansion processes, together with the temperature drops in vortex tubes using different working fluids, are presented in Table 1. It should be noticed that the two vortex tubes, from the experiments, differ in dimension and the temperature drops are average values instead of the lowest temperatures observed inside the tube. It can clearly be seen from Table 1 that, for the given conditions, the temperature variations of different gases during an isentropic expansion are significantly greater than that in both free expansion and Joule-Thomson expansion process. This agrees well with the discussions above, regarding equation 15 , that the isentropic expansion has the best cooling capability compared to other expansion processes under the same conditions. Note that the temperature of the ideal gas system remains constant in a free expansion; the small magnitude temperature variations in Table 1 are observed for real gases. Similarly, in a Joule-Thomson expansion process, a negligible temperature change occurs for the different gases, as seen in Table 1 . Therefore, the free expansion model and the JouleThomson model both predict a miniscule temperature drop during the expansion process under the given conditions. Comparing to the collected experimental temperature drops from vortex tube under the same conditions (last two columns in Table 1) it can be observed that the isentropic expansion is the only possible expansion process in the low pressure region within a vortex tube, due to its higher cooling capacity. As noted in previous research $[18,41]$, the lowest temperature within a vortex tube occurred in the central region near the injection

\begin{tabular}{|c|c|c|c|c|c|}
\hline $\begin{array}{l}\text { Temperature variation } \\
\text { (K) }\end{array}$ & $\begin{array}{l}\text { Isentropic Expansion } \\
T_{\text {Isentropic }}\end{array}$ & $\begin{array}{c}\text { Free Expansion } \\
\mathbf{T}_{\text {Free }}\end{array}$ & $\begin{array}{l}\text { Joule-Thomson } \\
\text { Expansion } \mathrm{T}_{\mathrm{JT}}\end{array}$ & $\begin{array}{l}\text { Vortex tube } \\
P_{\text {in }}=2 \text { bar [30] }\end{array}$ & $\begin{array}{c}\text { Vortex tube } \\
P_{\text {in }}=2 \text { bar [40] }\end{array}$ \\
\hline Air & -42.098 & -0.268 & -0.256 & -16.5 & -15.5 \\
\hline $\mathrm{O}_{2}$ & -42.095 & -0.271 & -0.277 & -16 & ------ \\
\hline $\mathrm{He}$ & -58.954 & -0.0111 & 0.099 & -21.5 & ----- \\
\hline $\mathrm{N}_{2}$ & -42.098 & -0.268 & -0.249 & ------ & ------ \\
\hline $\mathrm{CO}_{2}$ & -32.797 & -0.520 & -0.688 & ------ & ------ \\
\hline $\mathrm{Ar}$ & -58.752 & -0.444 & -0.377 & ------ & ------ \\
\hline $\mathrm{NH}_{3}$ & -34.546 & -0.648 & -0.879 & ------ & ------ \\
\hline
\end{tabular}
(low pressure region), which is apparently lower than the collected data illustrated in Table 1. Therefore, the maximum temperature drop within a vortex tube can be greater than that reported in the data and, therefore, closer to the temperature profile of an isentropic expansion process. Furthermore, the decrease in the flow velocity (cold flow),

Table 1: Temperature variation prediction for different expansion processes and temperature drop from a vortex tube at similar conditions. The negative sign denotes a decrease in temperature as the expansion occurs. 


\begin{tabular}{|c|c|c|c|c|c|c|c|}
\hline \multicolumn{8}{|c|}{ Maximum temperature drop (K) } \\
\hline & $\begin{array}{l}\text { Isentropic } \\
P=2 \text { bar }\end{array}$ & $\begin{array}{c}\text { Joule-Thomson } \\
\qquad \begin{array}{l}\mathrm{P}=2 \text { bar }\end{array}\end{array}$ & $P_{\text {in }}=1.5 \mathrm{bar}[30]$ & $P_{\text {in }}=2$ bar [30] & $P_{\text {in }}=3$ bar [30] & $P_{\text {in }}=7$ bar [31] & $P_{\text {in }}=3$ bar [33] \\
\hline Air & 42.098 & 0.256 & 10.5 & 16.5 & 26 & 39.5 & ------ \\
\hline $\mathrm{O}_{2}$ & 42.095 & 0.277 & 10.5 & 16 & 27 & 39 & ------ \\
\hline $\mathrm{He}$ & 58.954 & -0.099 & 13 & 21.5 & 34.5 & ------ & ------ \\
\hline $\mathbf{N}_{2}$ & 42.098 & 0.249 & ------ & ------ & ----- & 40 & 6.66 \\
\hline $\mathrm{CO}_{2}$ & 32.797 & 0.688 & ------ & ------ & ------ & ------ & 5.39 \\
\hline $\mathrm{Ar}$ & 58.752 & 0.377 & ------ & ------ & ------ & 53 & ------ \\
\hline $\mathrm{NH}_{3}$ & 34.546 & 0.879 & ----- & ------ & ----- & ----- & ------ \\
\hline
\end{tabular}

Table 2: Maximum temperature drop in the vortex tube and theoretical temperature drops.

which can leads to an increase in the fluid temperature, is another important factor for the difference in the temperature variations.

\section{Impact of fluid properties on the vortex tube cooling performance}

Considering the significant impact of gas properties on both the temperature variation in an adiabatic expansion process and the cooling performance of a vortex tube, the isentropic expansion model is further examined for different gases. The cooling performance or temperature drop of a vortex tube is a function of different parameters, including the geometrical parameters of the tube $\left(G_{\mathrm{vt}}\right)$, inlet $\left(P_{\text {in }}\right)$ and outlet conditions $\left(P_{\text {out }}\right)$, and the properties of the working fluid $\left(P_{\text {fluid }}\right)$. This function can be expressed as:

$$
\Delta T_{c}=f\left(G_{v t}, P_{\text {in }}, P_{\text {out }}, P_{\text {fluid }}\right)
$$

The maximum temperature drops in a vortex tube using different gases and the theoretical temperature drops are presented in Table 2. It can clearly be seen from the table that the maximum temperature drop from the vortex tube is dependent on the inlet pressure of the working fluid as well as the fluid properties. Note that different geometries of the vortex tube and different inlet parameters have been used in all these investigations listed in Table 2 . Therefore, in order to illustrate the effect of fluid properties, a normalized parameter, i.e., the temperature drop ratio $\left(\gamma_{F}\right)$ is used in this analysis. The normalized temperature drop can be defined as the ratio of the actual temperature drop $\left(\Delta T_{c, i}\right)$ to the temperature drop using air in the same situation $\left(\Delta T_{c, a i r}\right)$.

The remarkable characteristic of this ratio is that the temperature drops are equitable when different gases are used in the same vortex tube and at same operational conditions. This implies that the parameters " $G_{v t}, P_{\text {in }}$ and $P_{\text {out }}$ " for the recorded temperature drops are exactly the same; hence, their impacts can be removed from the function by this division. Then, only the effect of the fluid property on the cooling performance of a vortex tube is indicated by this ratio. Using the theoretical calculation and experimental data, the normalized temperature drop ratio is calculated using equation 17 and presented in Figure 2. As can be observed, the temperature drop ratios calculated based on the experimental data agree well with each other. This further indicates the reliability of the normalized temperature drop ratio in this analysis. Furthermore, an agreement between the experimental temperature drop ratio and theoretical results in an isentropic expansion process is also observed from the figure, which illustrates a strong correlation between the adiabatic isentropic expansion model and the cooling performance of a vortex tube. This correlation provides further support for the proposed hypothesis that the temperature drop in a vortex tube is mainly dominated by the isentropic expansion, and indicates the specific heat ratio of the working fluid is the primary factor that determines the cooling performance of a vortex tube.

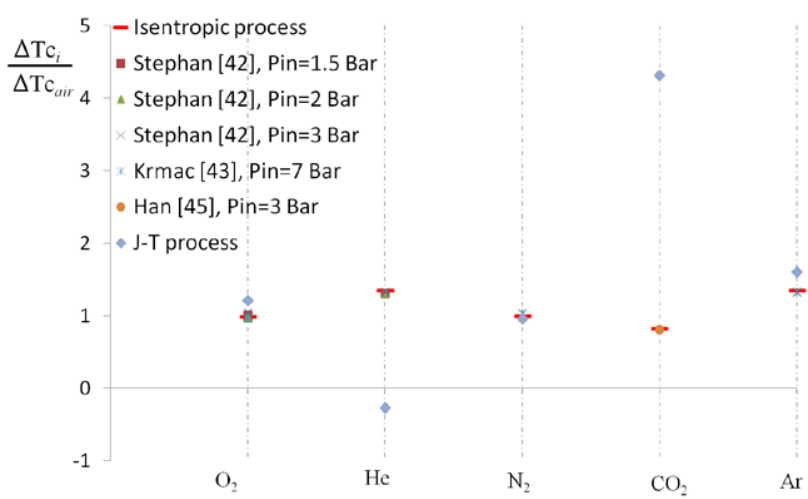

Figure 2: Temperature drop ratio of different gases in adiabatic expansions and vortex tubes.

\section{Discussion}

During the operation process of a vortex tube, the energy separation phenomenon also results in the temperature difference between the tube itself and the surroundings. More specifically, the cold and hot part of the tube is generally found having lower and higher temperature than the ambient air, respectively. Therefore, heat transfer occurs during the process, which has been reported in several experimental investigations [30,42-43]. The heat transfer from ambient surrounding to the cold part of a vortex tube leads to a decrease in the temperature drop of the cold flow. Similarly, the heat transfers from the hot part of the tube to the ambient air results in a decrease in the temperature rise of the hot exist. However, considering the dimensions of a commercial vortex tube (generally of the order of $100 \mathrm{~mm}$ in length and $10 \mathrm{~mm}$ in diameter) [40], the magnitude of velocity in the low pressure region near the injection can be as high as $200 \mathrm{~m} / \mathrm{s}$ [21,41-44]. This indicates that the expansion mechanism occurs in an extremely short timeframe. Hence, the heat exchange between the working gas and ambient surrounding could be neglected. Indeed, the impact of the heat transfer between the vortex tube and ambient surroundings has been shown insignificant in the abovementioned research. The employment of an isolating system resulted in an average 3.8 percent improvement of the vortex tube cooling performance $[30,42,43]$. Therefore, it is justifiable to claim that the adiabatic expansion process can be used to describe the same process inside a vortex tube, to some extent. In an isentropic expansion, the gas does positive work during the expansion process and its temperature drops. Within a vortex tube, the inner part of the compressed gas also does positive work on both the ambient surrounding and the peripheral part of the flow. The velocity profiles within a vortex tube have been reported by many researchers $[15,16,41,45]$. The flow mechanism near the injection of a vortex tube was observed as a force 
vortex with a large-magnitude centrifugal acceleration (in order of $10^{6}$ $\mathrm{rad} / \mathrm{s}$ in commercial tubes). Therefore, because of this large centrifugal acceleration, the central fluid exerts compression on the peripheral fluid. Energy is transferred towards the periphery via the work done by the central fluid, which contributes to the temperature drop of the cold flow within a vortex tube. It has also been noticed that the theoretical temperature variation in an isentropic expansion is greater than that in a vortex tube in a similar condition. This is mainly caused by three reasons, i.e., heat transfer from ambient air to the cold part of the tube, temperature gradient within a vortex tube and entropy increase of the working fluid. The impact of the heat transfer is discussed above. The temperature gradient within a vortex tube indicates the outlet temperature (cold end) is higher than the minimum temperature within the tube. Before being exhausted from the cold nozzle, gas at different temperatures is mixed together within the vortex tube. This mixture results in a reduction of the cooling performance of a vortex tube and, hence, leads to the different temperature variations in an isentropic expansion and in a vortex tube in similar condition. The increase of the system entropy in a vortex tube is mainly caused by the turbulent eddies in the central region of the tube. The turbulent flow behaviour has been reported in the investigations of the flow pattern in a vortex tube and was clearly shown by the turbulent intensity of the flow, particularly by the peak value of the intensity in the central area of the tube $[1,41,46]$. The increased entropy of the system also results in a loss of total energy, which has been noticed in previous investigations $[18,31,35,36,42,45,47-50]$. A free expansion process is typically achieved by allowing the gas to expand into a vacuum. Hence, in this process, no work is done by the gas. For the ideal gas, its internal energy remains constant, as well as its temperature. For a real gas, the temperature changes depending on the initial conditions. Clearly as discussed above, the work done by the gas expansion in a vortex tube and the comparison between the estimated temperature change in a free expansion and that in a vortex tube, imply that a free expansion clearly does not take place in a vortex tube. Finally, for a Joule-Thomson expansion process (isenthalpic expansion process), the gas expands via a valve or porous plug under steady state conditions and the enthalpy of the system keeps constant. However, the highly turbulent nature of the flow in the central region of a vortex tube and the decreased enthalpy of the exits provide negative evidence. Moreover, the velocity component of the flow, which is always neglected in a Joule-Thomson expansion, has significant impact on the vortex tube performance and must be included in analysis. From Figure 2, it can, furthermore,

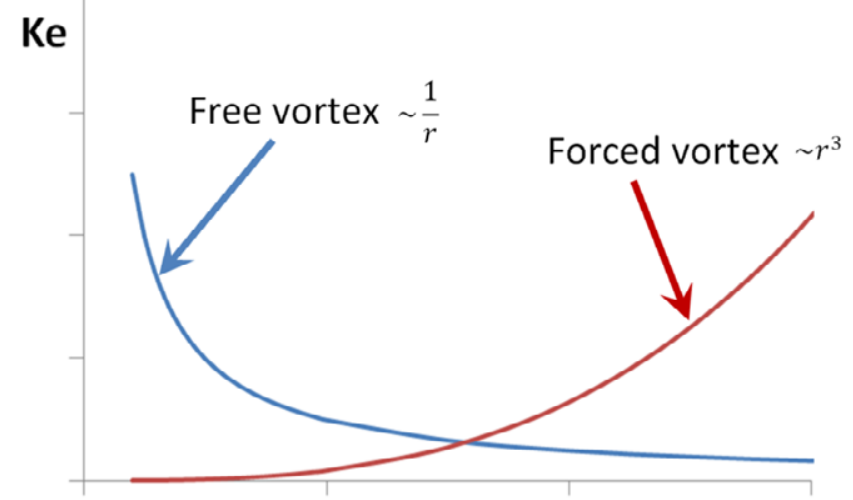

Figure 3: Non-dimensional kinetic energy level of a free vortex and a forced vortex. be observed that the cooling performance of Helium and Argon are superior to those of other working fluids; whereas, under the same conditions, the employment of Helium in a Joule-Thomson expansion process results in a temperature rise. The different cooling performance of the Joule-Thomson expansion process and the vortex tube, as shown in Figure 2, also suggests that the expansion process within a vortex tube should not be considered as an isenthalpic process. Additional to the expansion effect, the energy transferred outwards from the inner flow to the peripheral flow also contributes to the temperature drop in a vortex tube. The energy transfer is achieved through the transformation from a free vortex to a forced vortex. The kinetic energy level of a free vortex and a forced vortex is proportional to $1 / r$ and $r^{3}$, respectively (Figure 3); where, $r$ is the radius of the vortex. According to previous data $[15,18]$, in the central region of a vortex tube, the kinetic energy level of a free vortex is much higher than that of a forced vortex. Hence, in the transformation from a free vortex to a forced vortex, kinetic energy is transferred from the central flow to the peripheral flow and contributes to the separation process of a vortex tube. However, due to the lack of data, an overall quantitative analysis of the energy balance within a vortex tube is not available yet. An accurate energy analysis will, therefore, account for the kinetic energy transfer between different flow layers, the heat transfer between the ambient surrounding and the vortex tube, the effects of temperature gradient and the entropy increase of the working fluid due to turbulent eddies.

\section{Conclusion}

To obtain a better understanding of the separation phenomenon within a vortex tube, this manuscript reports an analytical investigation of the expansion process within the tube and the impact of the working fluid on the cooling performance of the tube. For a given set of conditions, the temperature variations in different expansion models are compared. With regard to the temperature drop within a vortex tube under the similar conditions, it was illustrated that the isentropic expansion model is the only plausible explanation of the expansion process inside a vortex tube. Further to this, the cooling performance of the vortex tube using different gases was also investigated and analyzed based on previous experimental results. A normalized temperature drop ratio is defined here to focus on the impact of the working fluid properties on the cooling performance of a vortex tube. A generally good agreement between the temperature drop ratios, calculated from both theoretical the experimental results, provides positive reinforcement for the isentropic expansion proposal as the primary expansion process inside a vortex tube. The heat capacity ratio of the working fluid is found to be the primary reason for the different cooling performances of different gases used in the vortex tube.

\section{References}

1. Xue $Y$, Arjomandi M, Kelso $R$ (2013) the working principle of a vortex tube International Journal of Refrigeration 36: 1730-1740.

2. Yalçin B, Özgür AE, Koru M (2009) the effects of various cooling strategies on surface roughness and tool wear during soft materials milling. Materials and Design 30: 896-899

3. Selek M, Tasdemir S, Dincr K, Baskaya S (2011) Experimental examination of the cooling performance of Ranque-Hilsch vortex tube on the cutting tool nose point of the turret lathe through infrared thermography method. International Journal of Refrigeration 34: 807-815.

4. Williams DT (2005) Ranque-Hilsch Vortex Tube for Refrigeration in Developing Communities. dissigno, San Francisco, CA USA.

5. Nellis G, Klein S (2002) the application of vortex tubes to refrigeration cycles. International refrigeration and air conditioning conference, USA.

6. Vranicar J (2001) A vortex contactor for carbon dioxide separations 
Citation: Xue Y, Jafarian M, Choudhry A, Arjomandi M (2015) The Expansion Process in a Counter-flow Vortex Tube. Fluid Mech Open Acc 2: 114. doi:10.4172/2090-8369.1000114

7. Ranque GJ (1933) Experiments on expansion in a vortex with simultaneous exhaust of hot air and cold air. J Phys Radium 4: 112

8. Liew R (2012) Temperature, Pressure and Velocity measurements on the Ranque-Hilsch Vortex Tube. Journal of Physics: Conference Series 395 012066 .

9. Khodorkov IL, Poshernev NV, Zhidkov MA (2003) Vortex tubes for gas heating, cooling, cleaning, drying, and separation. Khimicheskoe I Neftegazovoe Mashinostroenie 7: 24-27.

10. Kocabas $F$ (2010) Modeling of heating and cooling performance of counter flow type vortex tube by using artificial neural network. International Journal of Refrigeration 33: 963-972.

11. Dincer K (2008) Modeling of the effects of length to diameter ratio and nozzle number on the performance of counterflow Ranque-Hilsch vortex tubes using artificial neural networks. Applied Thermal Engineering 28: 2380-2390.

12. Dutta T, Sinhamahapatra KP, Bandyopdhyay SS (2010) Comparison of different turbulence models in predicting the temperature separation in a Ranque-Hilsch vortex tube. International Journal of Refrigeration 33: 783-792.

13. Zhou S, Meng Q, Liu D (2010) Numerical Investigations of Energy Separation into a Vortex Tube in Power and Energy Engineering Conference, Asia-Pacific.

14. Behera $U$ (2008) Numerical investigations on flow behaviour and energy separation in Ranque-Hilsch vortex tube. International Journal of Heat and Mass Transfer 51: 6077-6089.

15. Secchiaroli A (2009) Numerical simulation of turbulent flow in a Ranque-Hilsch vortex tube. International Journal of Heat and Mass Transfer 52: 5496-5511.

16. Xue Y, Arjomandi M, Kelso R (2012) Experimental study of the flow structure in a counter flow Ranque-Hilsch vortex tube. International Journal of Heat and Mass Transfer 55: 5853-5860.

17. Xue $Y$, Arjomandi M, Kelso R (2011) Visualization of the flow structure in a vortex tube. Experimental Thermal and Fluid Science 35: 1514-1521.

18. Xue Y, Arjomandi M, Kelso R (2013) Experimental study of the therma separation in a vortex tube. Experimental Thermal and Fluid Science 46: 175-182.

19. Yazid AA, Taha Z, Almanar IP (2010) A review of cryogenic cooling in high speed machining (HSM) of mold and die steels. Scientific Research and Essays 5: 412-427.

20. Yilmaz M (2009) A review on design criteria for vortex tubes. Heat and Mass Transfer 45: 613-632.

21. Eiamsa-ard S, Promvonge $P$ (2008) Review of Ranque-Hilsch effects in vortex tubes. Renewable and Sustainable Energy Reviews 12: 1822-1842.

22. Xue $Y$, Arjomandi M, Kelso R (2010) A critical review of temperature separation in a vortex tube. Experimental Thermal and Fluid Science 34: 1367-1374.

23. Kassner R, Knoernschild E (1948) Friction Laws and Energy Transfer in Circular Flow. Wright-Patterson Air Force Base, Technical Report F-TR-2198ND OH.

24. Arbuzov VA (1997) Observation of large-scale hydrodynamic structures in a vortex tube and the Ranque effect. Technical Physics Letters 23: 938-940.

25. Colgate SA Buchler JR (2000) Coherent transport of angular momentum. The Ranque-Hilsch tube as a paradigm. Annals of the New York Academy of Sciences 898: 105-112.

26. Kazantseva OV, Piralishvili SA, Fuzeeva AA (2004) Numerical simulation of swirling flows in vortex tubes. High Temperature 43: 608-613.

27. Ranque GJ (1934) Method and Apparatus for Obtaining from A Fluid under Pressure Two Outputs of Fluid at Different Temperatures 281

28. Liew R (2012) Maxwell's demon in the ranque-hilsch vortex tube. Physical Review Letters 109(5)

29. Shannak BA (2004) Temperature separation and friction losses in vortex tube. Heat and Mass Transfer 40: 79-785.

30. Stephan K. (1984) A similarity relation for energy separation in a vortex tube. International Journal of Heat and Mass Transfer 27: 911-920.

31. Krmac V, Uluer O, Dincer K (2010) An experimental investigation of performance and exergy analysis of a counterflow vortex tube having various nozzle numbers at different inlet pressures of air, oxygen, nitrogen, and argon. Journal of Heat Transfer 132(12).
32. Khazaei $\mathrm{H}$, Teymourtash AR, Malek-Jafarian M (2012) Effects of gas properties and geometrical parameters on performance of a vortex tube. Scientia Iranica 19: $454-462$.

33. Han X (2013) the influence of working gas characteristics on energy separation of vortex tube. Applied Thermal Engineering 61: 171-177.

34. Marshall J (1977) Effect of operating conditions, physical size and fluid characteristics on the gas separation performance of a Linderstrom-Lang vortex tube. International Journal of Heat and Mass Transfer 20: 227-231.

35. Volkan K (2009) Exergy analysis and performance of a counter flow RanqueHilsch vortex tube having various nozzle numbers at different inlet pressures of oxygen and air. International Journal of Refrigeration 32: 1626-1633.

36. Dincer K (2011) Experimental investigation of performance of hot cascade type Ranque-Hilsch vortex tube and exergy analysis. International Journal of Refrigeration 34: 1117-1124.

37. Polat K, Kırmacı V (2011) Determining of gas type in counter flow vortex tube using pairwise fisher score attribute reduction method. International Journal of Refrigeration 34: 1372-1386.

38. Polat K, Krmac V (2011) Application of the output dependent feature scaling in modeling and prediction of performance of counter flow vortex tube having various nozzles numbers at different inlet pressures of air, oxygen, nitrogen and argon. International Journal of Refrigeration 34: 1387-1397.

39. Moran MJ (2011) Fundamentals of engineering thermodynamics. N.J Wiley, Hoboken.

40. http://www.exair.com/vortextube/vt.

41. Gao C (2005) Experimental study on the Ranque-Hilsch Vortex Tube. J of cryogenics $45:$ 173-183.

42. Stephan K (1983) an investigation of energy separation in a vortex tube. International Journal of Heat and Mass Transfer 26: 341-348.

43. Promvonge $P$, Eiamsa-ard $S$ (2005) Investigation on the vortex thermal separation in a vortex tube refrigerator. Science Asia 31: 215-223.

44. Fröhlingsdorf W, Unger $H$ (1999) Numerical investigations of the compressible flow and the energy separation in the Ranque-Hilsch vortex tube. International Journal of Heat and Mass Transfer 42: 415-422.

45. Bruun HH (1969) Experimental Investigation of the Energy Separation in Vortex Tubes. Journal of Mechanical Engineering Science 11: 567-582.

46. Shamsoddini R, Khorasani AF (2012) A new approach to study and optimize cooling performance of a Ranque-Hilsch vortex tube. International Journal of Refrigeration 35: 2339-2348.

47. Markal B, Aydin O, Avci M (2012) Exergy analysis of a counter-flow RanqueHilsch vortex tube having different helical vortex generators. International Journal of Exergy 10: 228-238.

48. Saidi MH (1999) Exergy model of a vortex tube system with experimental results. Energy 24: 625-632.

49. Dincer K (2010) Experimental investigation and exergy analysis of the performance of a counter flow Ranque-Hilsch vortex tube with regard to nozzle cross-section areas. International Journal of Refrigeration 33: 954-962.

50. Eiamsa-ard S (2010) Experimental investigation of energy separation in a counter-flow Ranque-Hilsch vortex tube with multiple inlet snail entries. International Communications in Heat and Mass Transfer 37: 637-643. 